\title{
UNRECOGNIZED ORIGIN SIGNALS DISTURBING WATER-TUBES TILTMETERS MEASUREMENTS IN GEODYNAMIC LABORATORY OF SRC IN KSIAZ
}

\author{
Marek KACZOROWSKI \\ Space Research Centre, Polish Academy of Sciences, Bartycka 18A, 00-716 Warsaw, Poland \\ Corresponding author's e-mail: marekk@cbk.waw.pl
}

(Received February 2013, accepted June 2013)

\begin{abstract}
The harmonic oscillations of water level changes with several hundred nanometers amplitudes and $10^{-3}[\mathrm{~Hz}]$ frequencies are irregularly observed with the water-tube tiltmeter.

The effects are observed only by one of four gauges - the one closest to the entrance to underground. The other three gauges of water-tube tiltmeters are situated at the ends of the corridors and are significantly more distant from the entrance to the underground.

The atmospheric pressure signals affect level of water in the instrument by inverse barometric effect. The water-tube tiltmeters can register water level variations associated with inverse barometric effects, which were produced by air pressure signals until $10^{-5}[\mathrm{~Pa}]$ magnitude. The amplitude of harmonic oscillations of water level is significantly large and amounts to $1 / 10$ of tidal amplitude, that is 300 to 500 nanometers, which corresponds to $5 \times 10^{-3}[\mathrm{~Pa}]$ of air pressure variations.

Harmonic oscillations appear irregularly and their time of duration changes from few days until several weeks. The length of atmospheric wave of $10^{-3}[\mathrm{~Hz}]$ frequencies is of $1000[\mathrm{~km}]$ and cannot be explained by any phenomenon from the outside surroundings or the inside of the underground. Resonance frequencies of air column in the hundred meters long underground corridors are of the order of single $\mathrm{Hz}$.

Absence of similar signals in measurements from the other three gauges suggests that the harmonic signals are not propagated through the Earth's solid body but through the atmospheric medium.

The observed micro-vibrations of air pressure are very low infrasounds which can be produced in large dimension space of thousand kilometers size.

The open question is what is the origin of micro-vibrations registered by the water-tube tiltmeter.

KEYWORDS: Earth tides, water-tube tiltmeters, disturbances of plumb line variations, infrasound measurements, natural sources of micro-vibrations, atmospheric resonance, interaction between ocean-atmosphere and ionosphereatmosphere
\end{abstract}

\section{INTRODUCTION}

Since 2003 in Geodynamic Laboratory of SRC (LG) two water-tube tiltmeters (WT) are permanently at work (Kaczorowski, 2006B). The tiltmeters consist of two disconnected hydrodynamic systems, the main elements of which are perpendicular tubes 65.24 and $93.51 \mathrm{~m}$ long. The tubes are located precisely horizontally at azimuths $-121.4^{\circ}$ and $-31.4^{\circ}$ respectively. Applied construction of hydrodynamic systems preserves free surface of water on the whole length of the tubes. Water occupies half space of the tubes approximately. At every four meter length inside the tubes there are stoppers with little holes. The holes are under water and their diameters were chosen appropriately on the basis of experiments. The stoppers separate each of tubes on dozen or so sections. In every section, at the top of the tubes there is one hole connecting the inside of tubes with the space of corridors. The holes at the top of the tubes assure rapid compensation of differences of the air pressure inside the tubes and their surroundings. The applied construction minimizes the noises, causing compensation of air pressure outside the tubes and not directly above the water surface.
Separation of the tubes into sections works as lowpass filter which limits the observed signals to ca. $<1 / 10 \mathrm{~Hz}$ frequency. Hydrodynamic systems assure perfect damping of high frequency water oscillations caused by earthquakes. This was proved during Sumatra-Andaman earthquake - 2004, Tohoku-Chiho Taiheiyo-Oki, Japan-2011 and during other strong seismic events (Kaczorowski, 2006A). Hydrodynamic systems of tiltmeters were assembled in straight horizontal corridors of cross section equal to 16 square meters (Fig.1). Large sizes of corridors cause that the horizontal gradient of air pressure generally is compensated in tunnels in a few seconds and therefore does not affect water level in instruments.

\section{SOME RESULTS OF MEASUREMENTS OF PRESSURE, TEMPERATURE, AND HUMIDITY (TPH) IN LG}

Indications of TPH system play important role in further discussion presented in this paper. In 2007 LG was equipped with system of 26 gauges of pressure, temperature, and humidity (TPH). The gauges are distributed in the underground in surrounding of sensors of geodynamic instruments. TPH system 


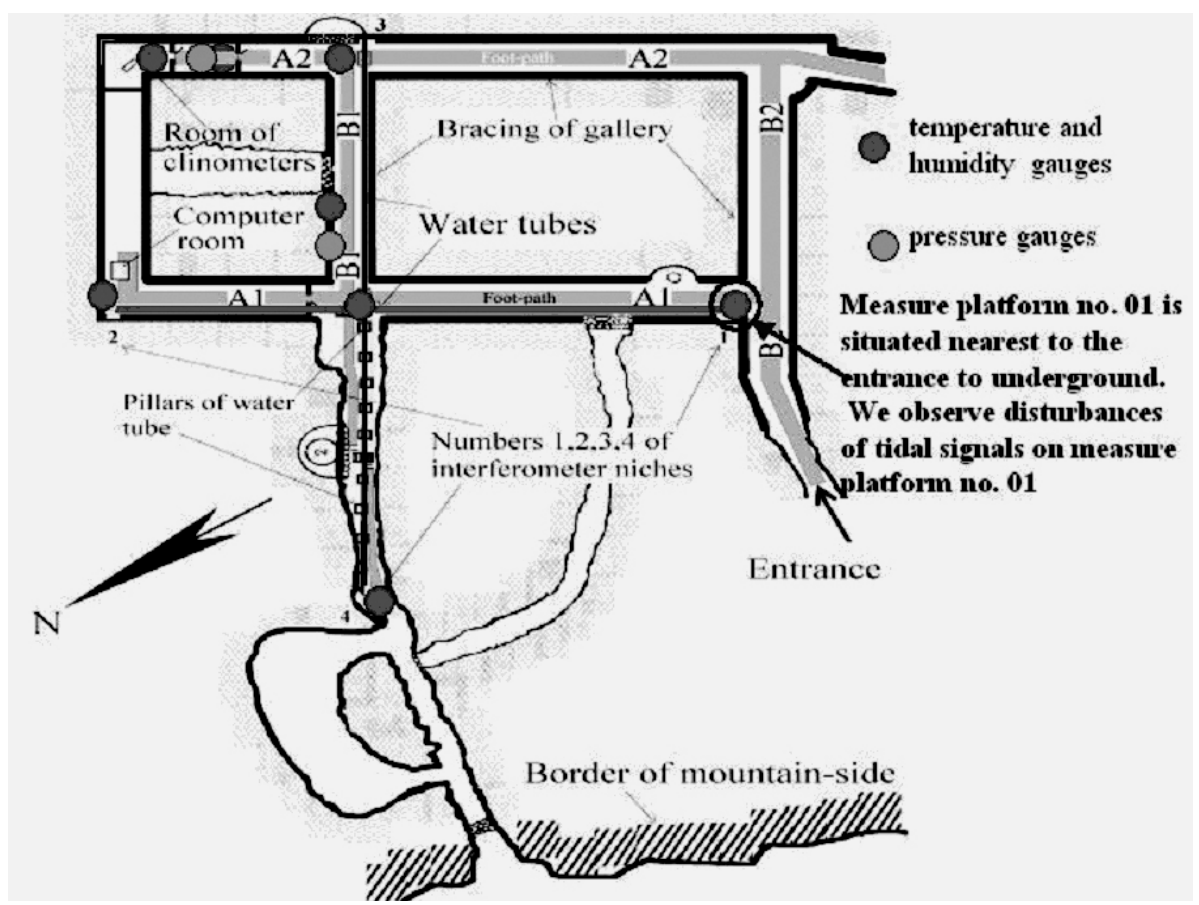

Fig. 1 Plan of the underground with situation of WT tiltmeters, TPH system and locations of measure platform no. 01 where disturbing signals are observed.

registers variations of parameters every fifteen seconds with resolution of $0.01^{0}[\mathrm{C}]$ - temperature, $0.1 \%$ - humidity and about $0.2[\mathrm{hPa}]$-pressure.

Since 2010 we additionally possess the meteorological station (METEO) associated with GNSS station on the house of laboratory. The METEO station is equipped with permanent TPH system with resolution of $0.01^{0}[\mathrm{C}]$ - temperature, $0.1 \%$ - humidity and $0.1[\mathrm{hPa}]$-pressure registering every minute. Between the surface and the underground TPH systems there is about 52 meter difference in height. However, METEO plot of pressure variations is above the pressure plots (PG and SR) from the underground (Fig. 2). The pressure plots from the underground are vertically shifted as well as are incorrectly shifted in relation to METEO plot because PG and SR gauges have never been absolutely calibrated. Locations of gauges in the underground were shown in Figure 1. The pressure gauges are inside the gravimetric pavilion (PG) and at the crossing of tubes (SR) which are distant about 50 meters. The pressure gauge inside the gravimetric pavilion $(\mathrm{PG})$ is separated from the space of corridor by two doors. Additionally, between the corridor with SR gauge and the corridor with gravimetric pavilion there is a door. These obstacles as well as imperfection of gauges are the reason of small differences between PG and SR plots (Fig. 2).

The capacity of underground amounts to 6000 cubic meters approximately, and total surface section of holes to the outside were evaluated by us as dozen or so square decimeters only. We expected tens of minutes lag between pressure accommodation in the underground to the outside pressure. Therefore the results of measurements were surprising. During the depression the minimum of plots of METEO and underground gauges were shifted several minutes only (Fig. 2). The gauges located in the underground inside gravimetric pavilion (PG) and at the crossing of tubes (SR) registered time shift of minimum of pressure close to the interval of sampling. This result is in accordance with the WT measurements. The results of WT measurements indicate that compensation of air pressure in the underground corridors occurs very fast (vanishing of horizontal pressure gradient), which prevents inverse barometric effects.

\section{MECHANISM OF WT MEASUREMENTS DISTURBANCES CAUSED BY ATMOSPHERIC EFFECTS}

From the beginning of work of the WT tiltmeters we observed that levels of disturbances of tidal signals registered by the tiltmeters significantly differ between measurement platforms no. 02, 03, 04 and platform no. 01 (Fig. 1). The amplitude of disturbing signal is two orders of magnitude greater on platform no. 01 than on platforms no. 02, 03, and 04 . Furthermore, platform no. 01 is situated closer to the entrance to underground than the other three platforms. Absence of similar signals on the other three platforms as well as location of platform no. 01 (Fig. 1) made it possible to conclude that the disturbing signals are propagated through the atmosphere medium and not the solid body of lithosphere. 


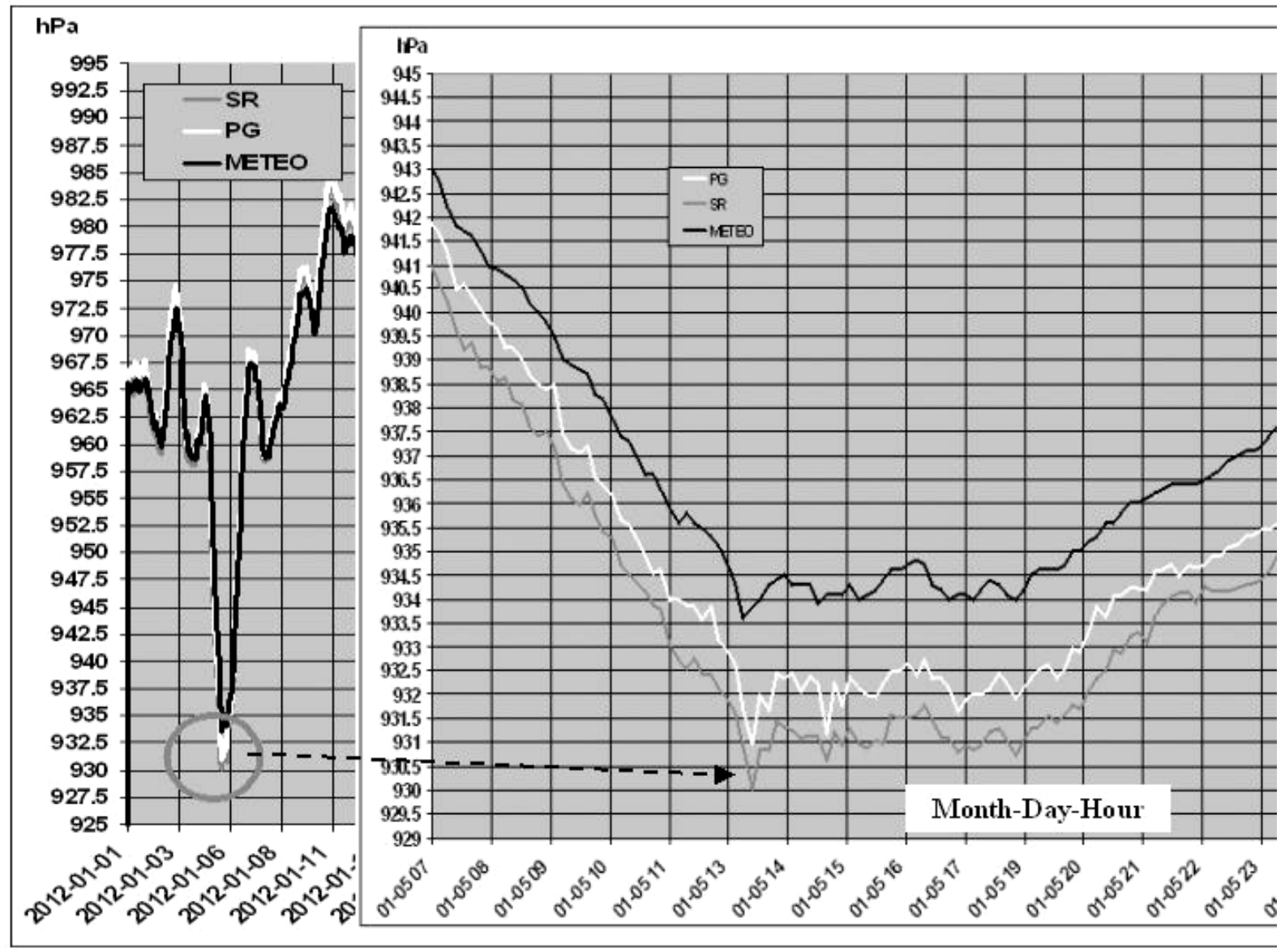

Fig. 2 Plots of pressure variations registered by the three gauges METEO, SR and PG dutiny the strong depression in January of 2012.

All signals such as tidal, free oscillations of the Earth, seismic or aperiodic or systematic and propagated through the lithosphere are simultaneously registered on all four measure platforms of WTs (Kaczorowski, 2004). The atmospheric pressure signals affected water level in the instruments by the inverse barometric effect. Sensitivity of interference gauges of WT is close to a single nanometer which corresponds to $10^{-5}[\mathrm{~Pa}]$ (Kaczorowski, 2006B).

Therefore the WT tiltmeters are at the same time microbarometers which are able to register the air pressure microvibrations until $10^{-5}$ [Pa] magnitude (Posmentier, 1967). However, the inverse barometric effect is seldom observed in the WT tiltmeters registrations (Chapter 5). To produce the inverse barometric effect suitable time of duration of the horizontal pressure gradient along the tubes of tiltmeters is necessary. If the duration time is shorter than the time of outflow of water, the inverse barometric effect does not appear. The time necessary for the outflow is in general longer than the time of pressure compensation in the corridors and disappearing of horizontal gradient (Chapter 1). Therefore only strong, rapid and long duration effect of pressure variations is appropriate for generation of inverse barometric effect (Fig. 7).

\section{REGISTRATION OF STRONG DISTURBANCES OF WT MEASUREMENTS ON 18TH TO 20TH JUNE, 2010}

On 18th to 20th of June 2010 we observed very strong around-tidal oscillations of water level. The amplitude of the disturbance achieved $10 \%$ of tidal amplitude. The effect was registered on measurement platform called 01 close to the entrance to underground (Fig. 1).

On the opposite end of the tube such disturbances did not appear (Fig. 3). The strongest disturbances appeared during the depression just after minimum of air pressure. Temperature variations at this time were small and amounted to few hundredths degree.

We extract disturbing signals in fourth steps. In the first step we determine difference of rough signal and ephemerid tide in the tube. The tidal ephemerid was determined on the basis of ten years long series of WT observations processed with help of "Analize" program which belongs to ETERNA packet (Wenzel, 1996).

In the second step two second order polynomial splines of one minute sampling and of 120 minutes sampling were constructed on the basis of difference of rough signal and ephemerid. The 120 minutes 


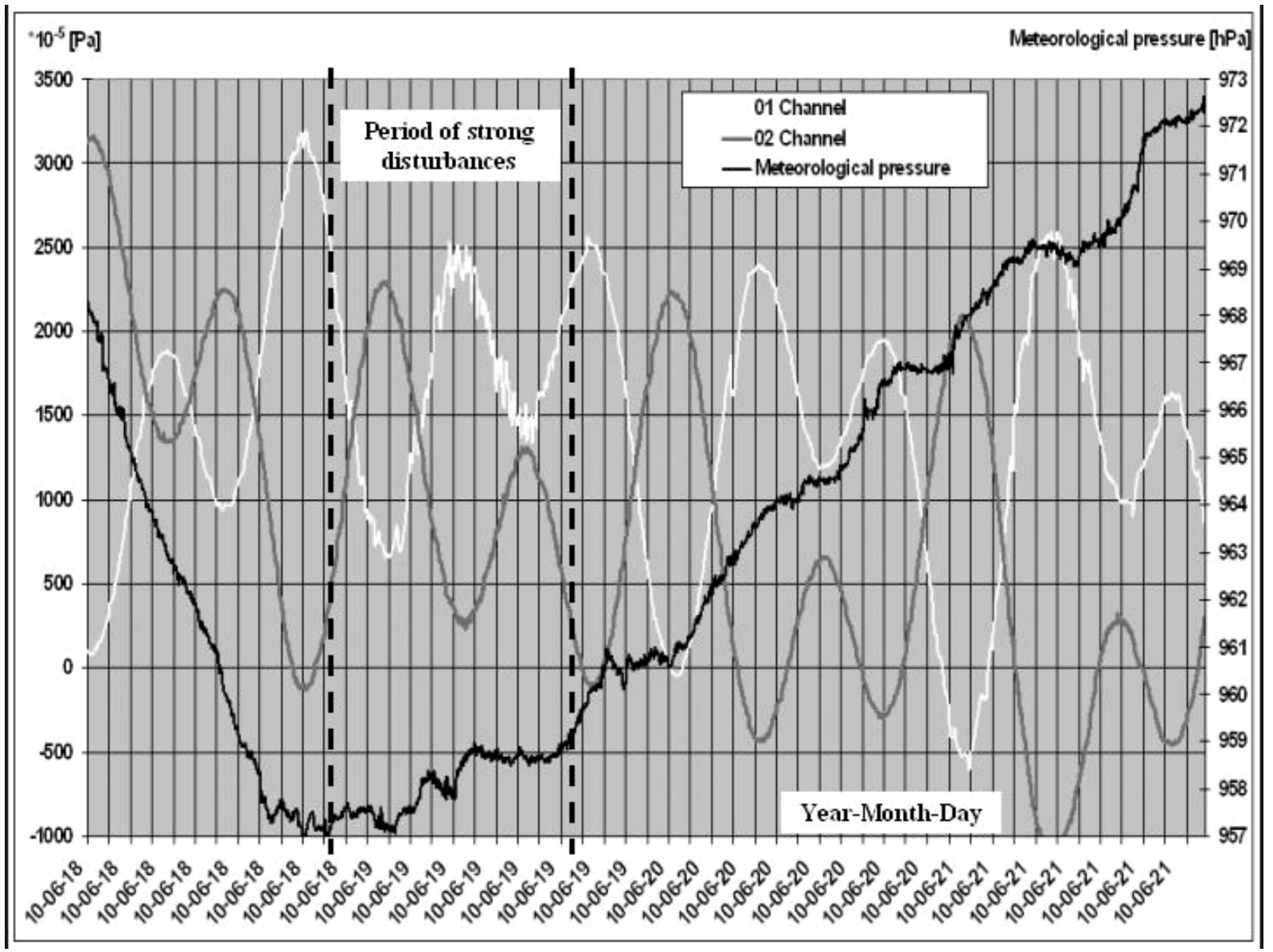

Fig. 3 Four days long plots of tidal measurements and pressure changes during strong disturbances.

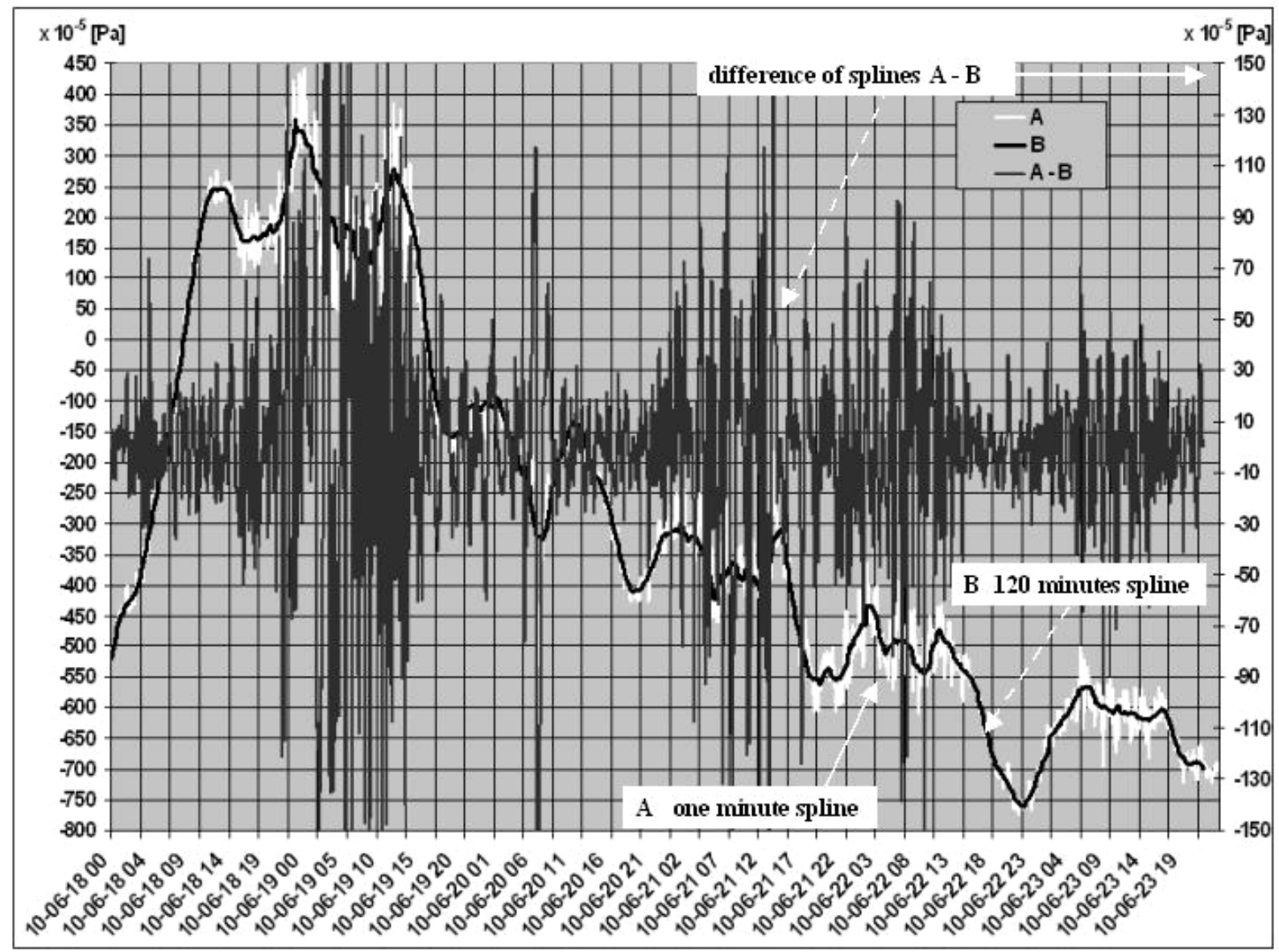

Fig. 4 Plots of one minute and 120 minutes sampling splines and their difference. 


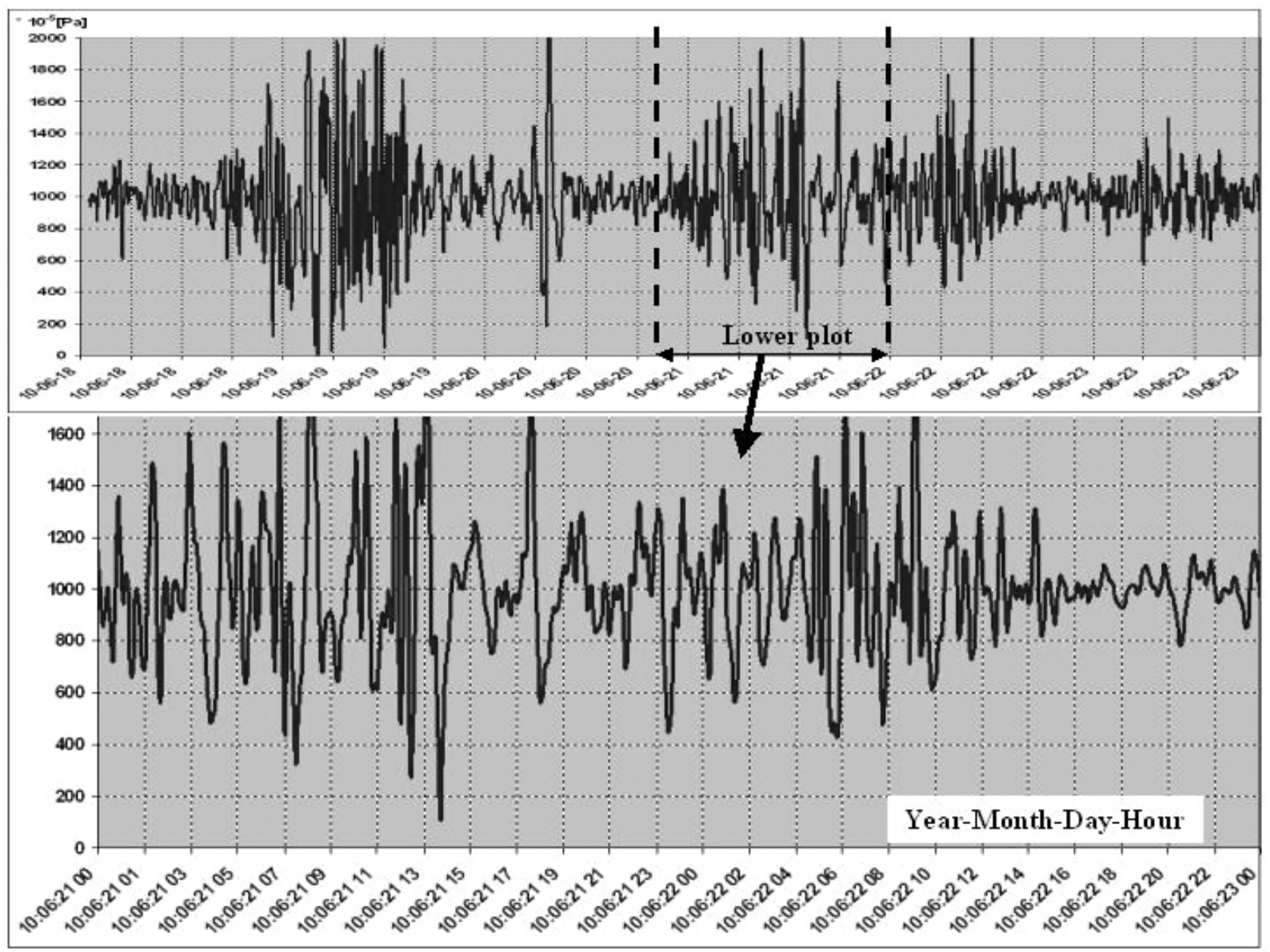

Fig. 5 Plots of disturbing signals after the lowpass filtration.

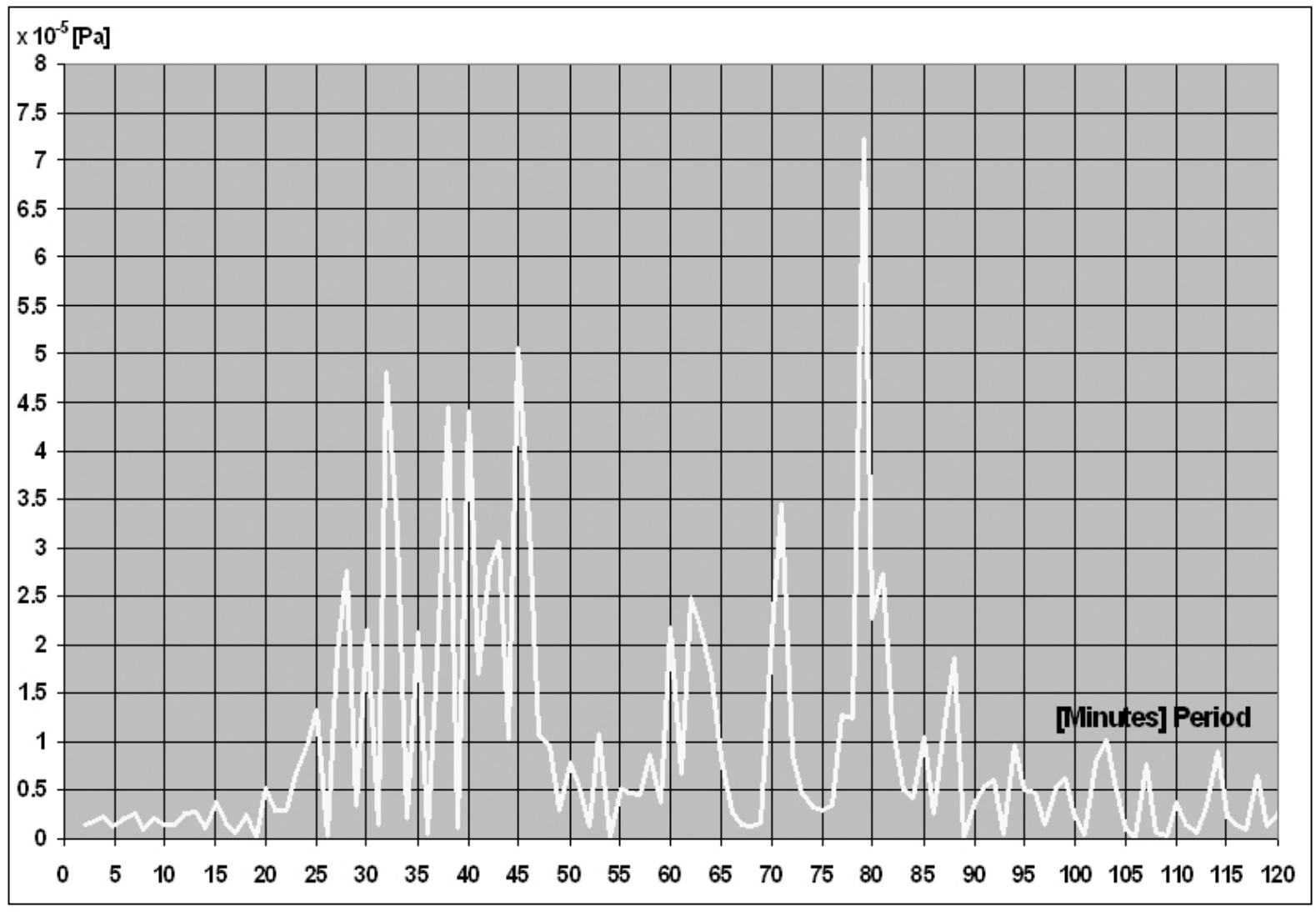

Fig. 6 Plot of power spectrum determined on the basis of records from the 18th to 20th of June 2010. 


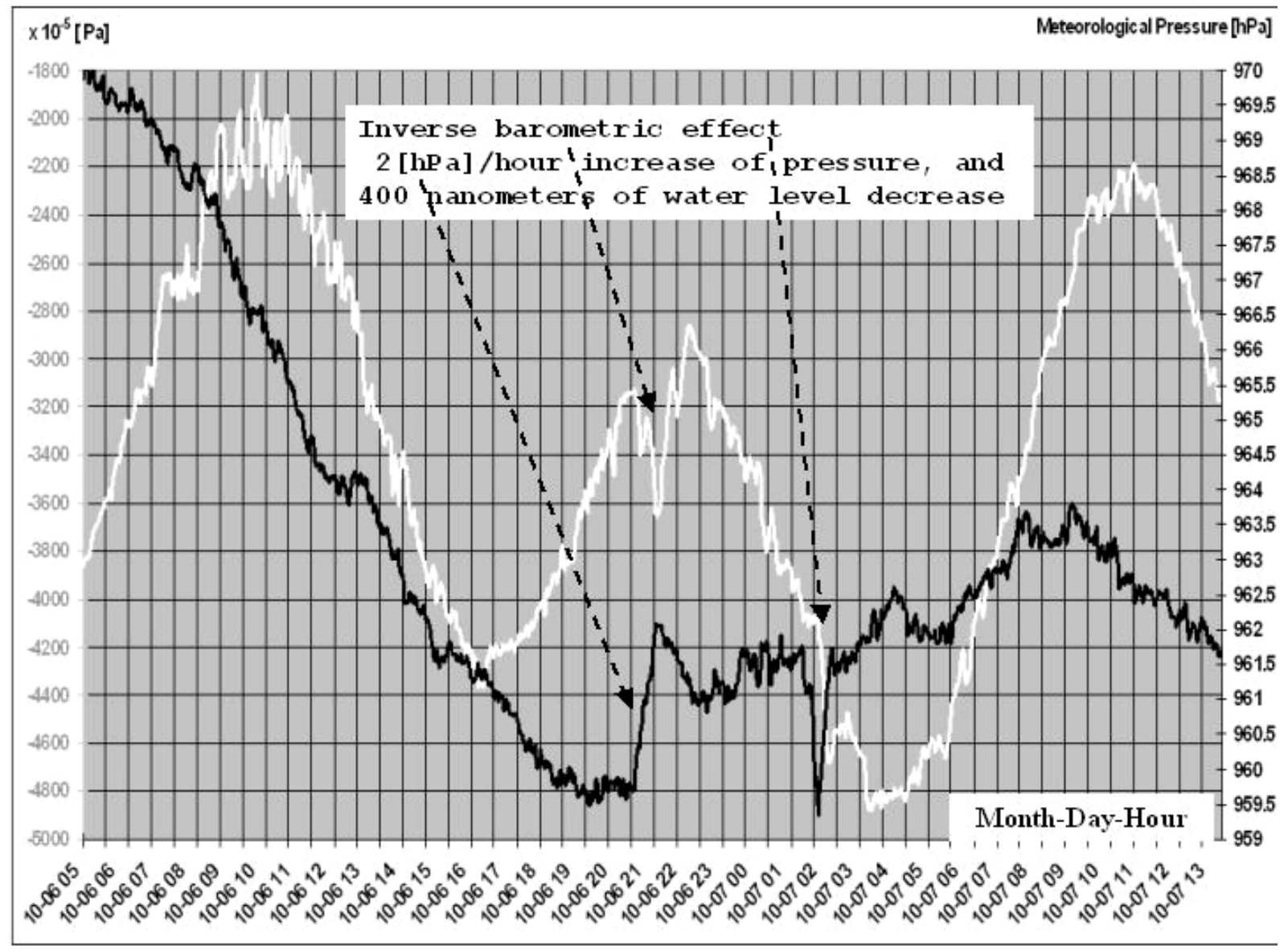

Fig. 7 Two-days long plots of air pressure variations and disturbances of tidal signals from the 6th and 7th October 2011.

sampling spline is a long period or systematic trend, and one minute sampling spline contains the disturbing signals as well as high frequency signals. In the third step we determined difference of splines of one minute and 120 minutes sampling. This difference contains disturbing signals and high frequency signals. In the fourth step difference of splines was lowpass filtrated, which considerably reduced contribution of short-period signals. For filtration we applied 5 minutes lowpass filter N60S5M01.NLF available for 60 second sampling data. The applied filter belongs to ETERNA packet (Wencel, 1996). Disturbing signals after filtration were presented in Figure 5. The plot of disturbing signal resembles superposition of harmonics (Fig. 5). The results of Fourier analysis confirm that the disturbing signals are of harmonic character (Fig. 6). The plot of power spectrum presents dozen or so harmonics which are separated into two groups with around 80 and 40 minutes periods.

\section{REGISTRATION OF STRONG DISTURBANCES OF WT MEASUREMENTS ON THE 6TH TO 7TH OCTOBER 2011}

During the days of 6th and 7th October 2011 the gauge on measuring platform no.1 (Fig. 1) registered large non-tidal oscillations of water level. In this event the magnitude of oscillations achieved $15 \%$ of tidal amplitude. Similarly to the previous event we did not observe such signals on the opposite end of the tube. The plot of tidal signals strongly disturbed by harmonic oscillations was shown in Figure 7 together with the plot of air pressure variations from the 6th and 7 th October 2011. On the days of 6th and 7th October, beside strong disturbing harmonic oscillations there occurred two events of inverse barometric effects, which additionally deformed tidal undulations. The inverse barometric effects appear in special conditions when pressure variations are enough strong and rapid to overtake the process of pressure compensation in the underground. Both effects, i.e. disturbing oscillations as well as inverse barometric effects, occurred during the specific phase of atmosphere in the time of deep depression.

Changes of pressure and temperature around the 6th and 7th October 2011 epoch are shown in Figure 8. During eleven days the pressure variations reached $25 \mathrm{hPa}$ and its variability was especially rapid in minimum of depression, when effects of disturbing oscillations were detected. In the whole interval the temperature was stable and slightly fluctuated with amplitude of $0.02^{0}[\mathrm{C}]$. Simultaneous appearance of 


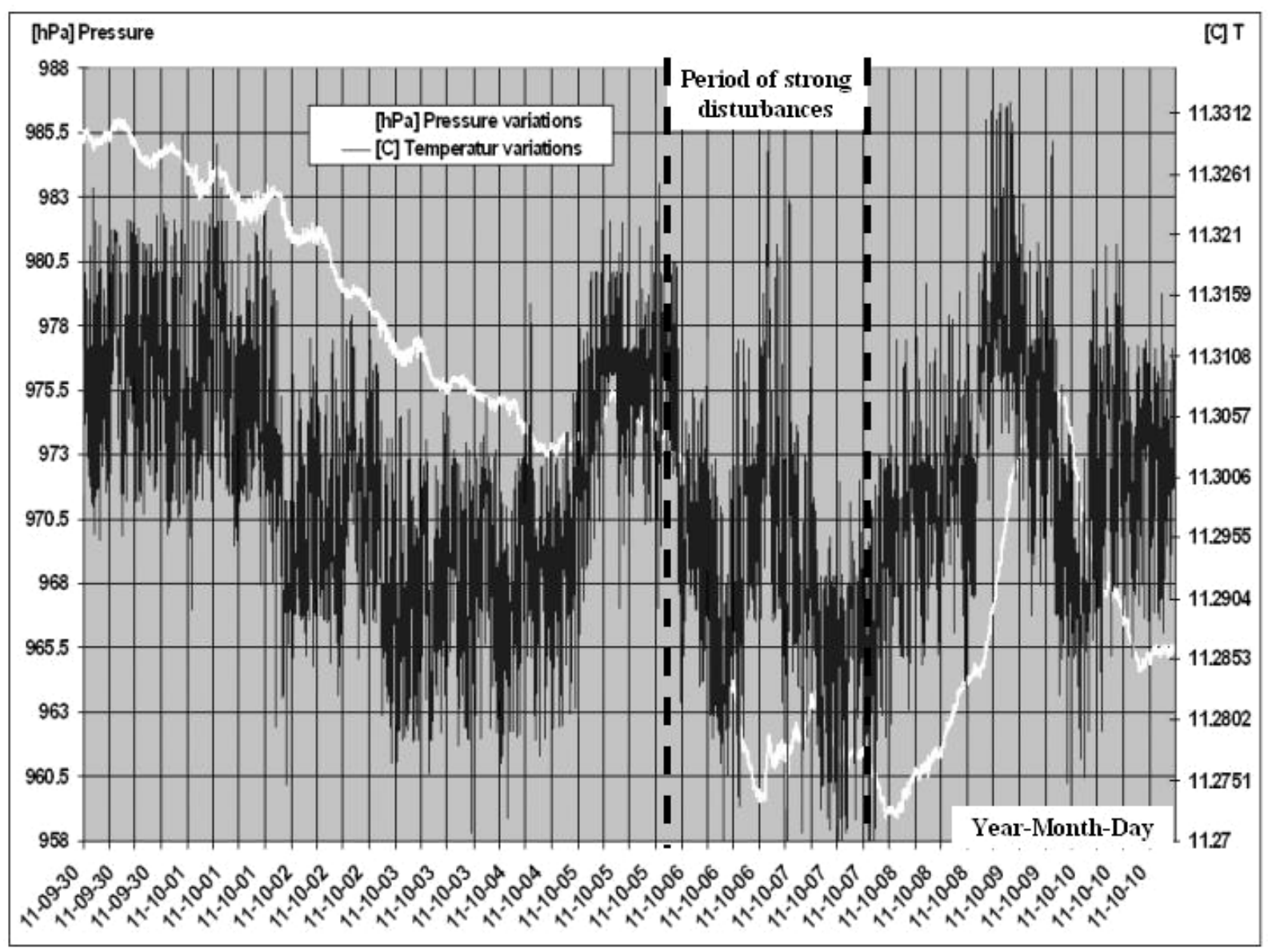

Fig. 8 Eleven days long plots of temperature and air pressure variations from around the epoch of 6th and 7th October 2011 of strong harmonic disturbances of tidal signals .

both effects, the inverse barometric and disturbing oscillations provided a good opportunity to investigate how the extreme local pressure variations interact with the disturbing harmonic signals.

For elaboration of raw observations we apply the method described in detail in Chapter 4. After application of the lowpass filtration we obtained series of dozen or so regular harmonic presented in Figure 9. In this event the harmonic signal consists of two groups of ca. 60 minute periods and 35 minute periods, similarly to the previous event (Figs. 10 and $6)$.

Comparing of the plot of harmonic oscillations (Fig. 9) to the plots of pressure and tidal signals (Fig. 7) we found coincidence between duration of inverse barometric effects and magnification of amplitude of harmonic signals. When the local pressure was a slower variable or it became smoother, then the harmonic oscillations became more regular (Fig. 9).

\section{REGISTRATION OF STRONG DISTURBANCES OF WT MEASUREMENTS ON 3RD TO 4TH MARCH 2012}

We studied about ten events of harmonic signals which occurred in the epoch 2010-2012. The event from the 3rd and 4th March 2012 significantly differs from the previous events (Chapters 4 and 5).

This event did not occur during deep depression in minimum of pressure but at the moment when the air pressure changed only slightly (Fig. 11). This example contradicts the thesis that events of harmonic signals are associated with regional atmospheric depressions. In Figure 11 we can notice that harmonic oscillations of the disturbing signals are independent from the local and meteorological origin air pressure micro oscillations.

Small and high frequency oscillations are visible in the plot of meteorological pressure only while the atmosphere is cooled down. As in the previous events the power spectrum of disturbing signals consists of dozen or so harmonics separated into two groups of around 55 and 75 minute periods (Fig. 12).

\section{CONCLUSIONS}

Preliminary investigations of the disturbing signals of WT tidal measurements provide us with several observations.

We found that the disturbing signals are propagated in the atmospheric medium. Possibility of propagation of disturbing signals through lithosphere in the Earth's solid body was excluded on the account 


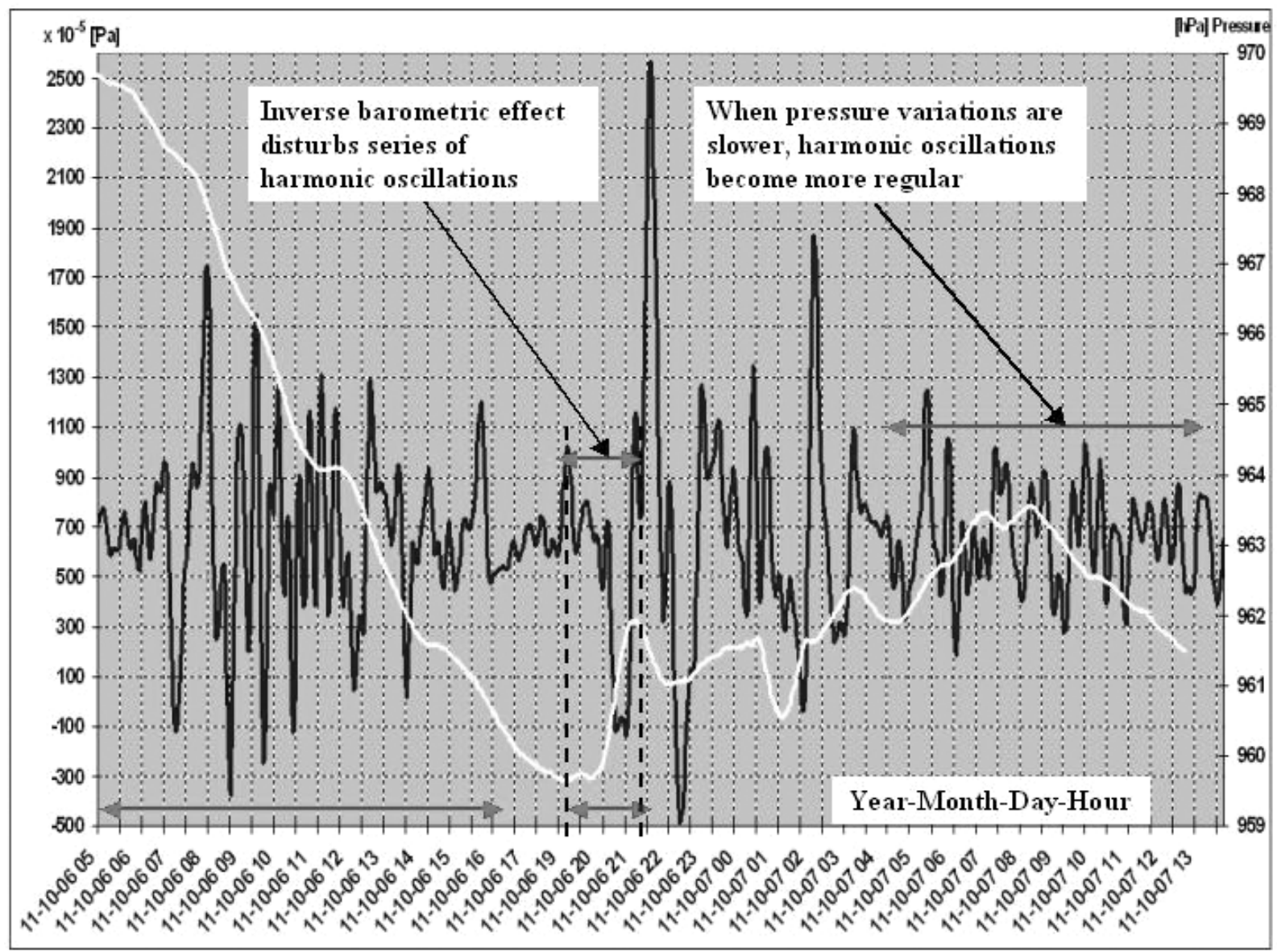

Fig. 9 Plots of disturbing signals after lowpass filtration and pressure changes in epoch 6th to 7 th October 2011.

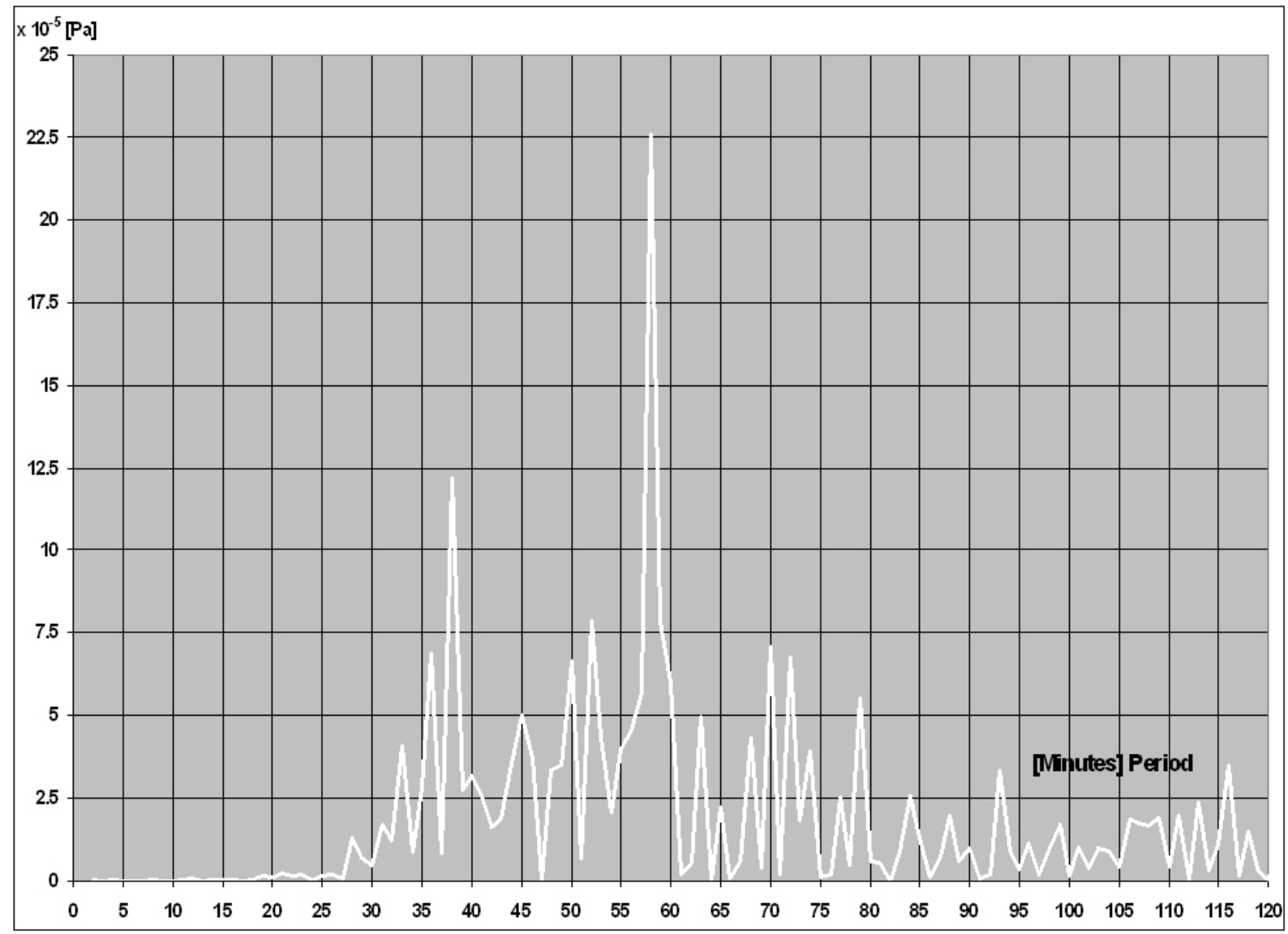




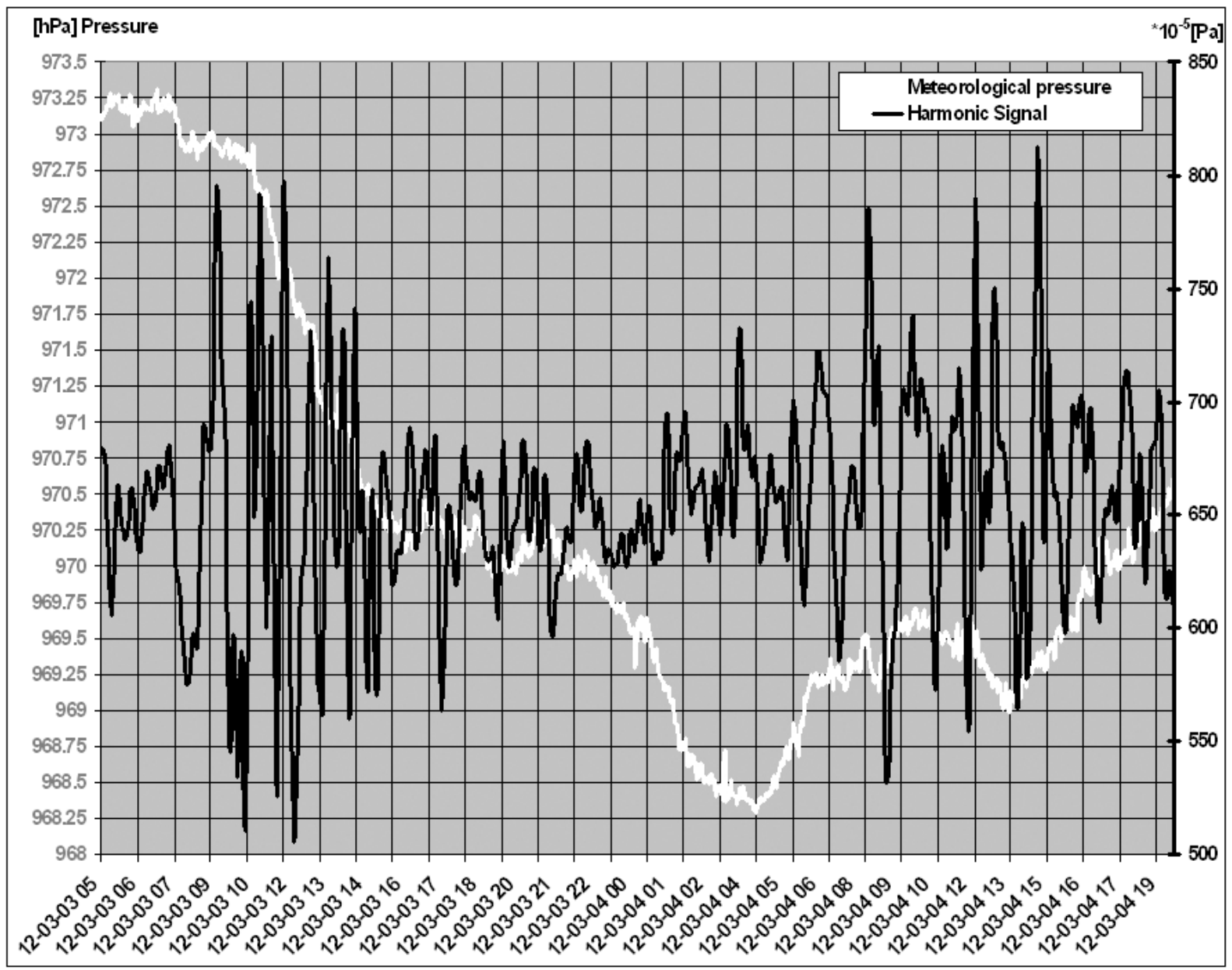

Fig. 11 Plots of disturbing signals after the lowpass filtration and pressure changes in epoch of 3rd and 4th March 2012.

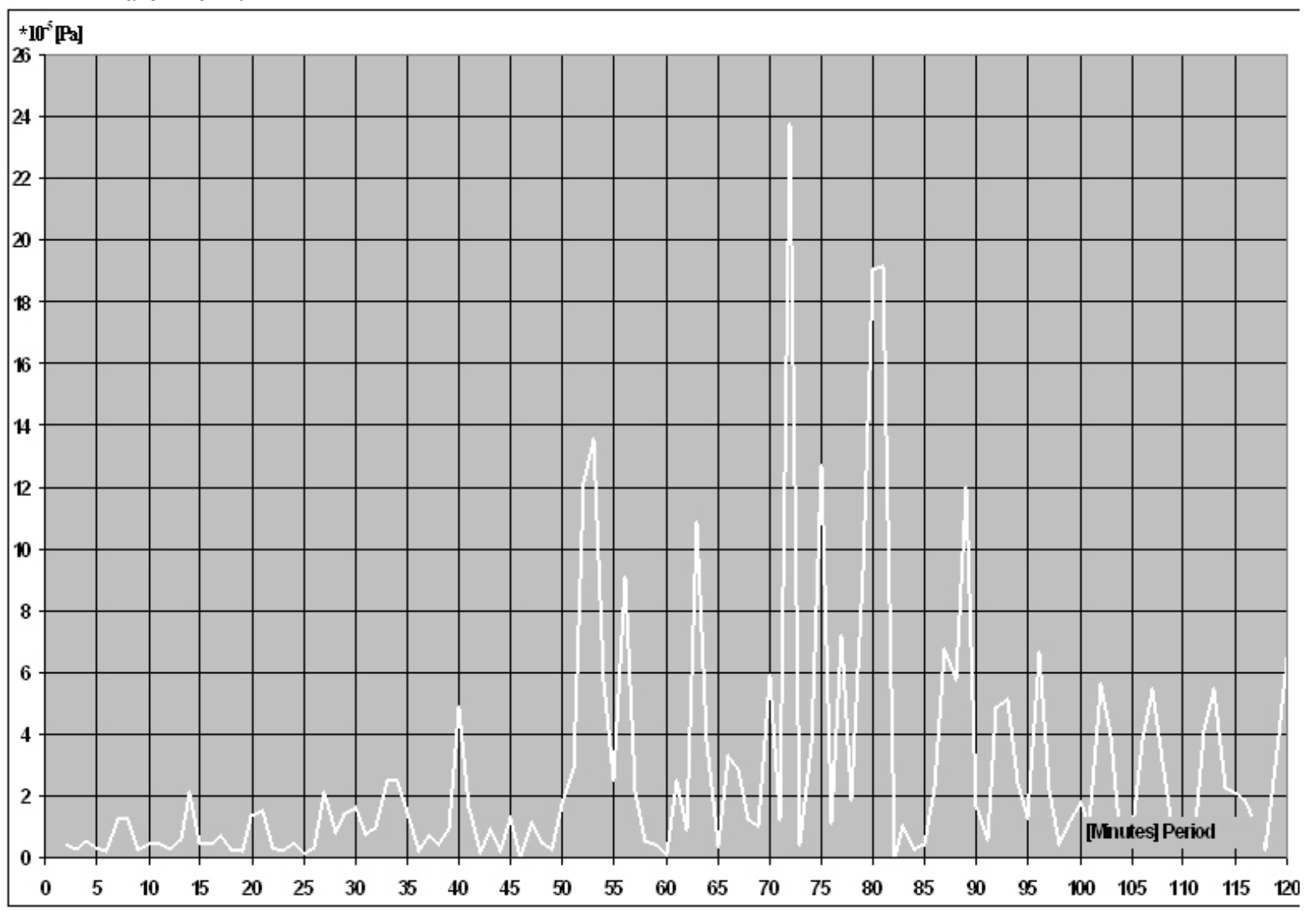

Fig. 12 Plot of power spectrum determined on the basis of record from epoch 3rd to 4th March 2012. 
of absence of these signals on measurement platforms named 02, 03, and 04 of WT tiltmeters (Fig. 1). The fact that only the gauge no. 01 which is close to the entrance to underground registers disturbances additionally authenticates relations between atmospheric effects and the observed phenomenon.

In such a case the only mechanism of transfer of the disturbing signals from the atmosphere to the hydrodynamic system of WT is inverse barometric effect.

Disturbing signals consist of a dozen or so harmonic modes of 10 to 100 minute periods $\left(10^{-3} \mathrm{~Hz}\right)$ (Figs. 5, 9, 11) the amplitudes of which depend on selected fragment of data (Figs. 6, 10, 12).

Therefore the disturbing signals are very low infrasounds with variable amplitudes of harmonic components.

Harmonic characteristics of signals suggest existence of resonance phenomenon which is the source of these signals. Taking into account velocity of propagation of infrasounds in atmosphere as about $330 \mathrm{~m} / \mathrm{sec}$ and $10^{-3} \mathrm{~Hz}$ frequency we expect infrasound waves of one thousand kilometers length.

Such waves can be produced in large dimension space of thousand kilometers size only. Very low frequency of phenomenon enabled us to remove speculations on the sources of infrasounds located inside the underground laboratory or in its surroundings.

Impossible to explain are also the instrumental reasons of phenomenon of $10^{-3} \mathrm{~Hz}$ frequency. Anthropologic origin of infrasounds is well known and documented. Microvibrations of $1 \mathrm{~Hz}$ to $5^{*} 10^{-2} \mathrm{~Hz}$ range are observed in various technical devices. Special devices for generation of microvibrations make it possible to obtain signals of maximally low frequency of $10^{-2} \mathrm{~Hz}$ order. Generally, very low infrasounds are difficult to be produced artificially because the sizes of devices generating infrasounds are much smaller than their wavelength and therefore the process of infrasounds generation is very ineffective.

Therefore looking for reasons of very low microvibrations we ought to concentrate on natural sources (Benioff and Gutenberg, 1939). There are known several natural mechanisms of generation of extremely low infrasounds. Very low infrasounds of $10^{-3}[\mathrm{~Hz}]$ frequency appeared in effect of interaction between the ionosphere and upper atmosphere.

Acoustic waves in the upper atmosphere are associated with temporal and spatial electron density variations in the ionosphere (Hoult, 1969). Possibility of generation of ion sound waves in the topside low latitude of ionosphere was reported by (Huba, Joyce, scientific letter).

Other sources of low frequency signals are seismoacoustic phenomena associated with seismic activity on the Earth. Low frequency oscillations of the Earth surface are propagated to the atmosphere in the form of infrasounds. There is very interesting example of seismoacoustic waves which are of nearly identical spectrum, as microseisms propagated in the Earth's solid media (Ponomarev and Sorokin, 1996).

One of the sources of low infrasound is the ocean - atmosphere interaction (Donn and Naini, 1973). Of special importance are two mechanisms associated with standing waves: deformation of sea topography and altering gravity of standing waves inside the ocean medium.

Which of the presented phenomena is a reason of harmonic signals observed by WT is not known but the most probable is the ionosphere and atmosphere interaction because of their extremely low frequency $10^{-3}[\mathrm{~Hz}]$.

There is a serious problem of why the infrasound signals with magnitude $10^{-3}[\mathrm{~Pa}]$ of harmonic characteristics and tens of minutes duration can disturb registration of WT tiltmeter. The inverse barometric effects are seldom observed in the WT tiltmeter registrations (Chapter 5). Pressure variations are able to produce the inverse barometric effect only in the case when these variations are strong and rapid enough to overtake the process of pressure compensation in the underground. In Figure 7 we can see that the large and fast meteorological pressure variations equal to about $2[\mathrm{hPa} /$ hour produced inverse barometric effect of $4 * 10^{-3}[\mathrm{~Pa}]$ only. The proportion between amplitude of meteorological pressure variations and water level variations is $5 * 10^{4}$ (Fig. 7). Therefore the meteorological pressure variations during the event from 6th and 7th October 2011 almost perfectly underwent compensation. The coefficient of compensation for this event amounts to $99.99 \%$. Therefore the WT tiltmeter registered only a very small residuum of meteorological pressure signals. In this event signals of microvibrations harmonic infrasounds produced the same magnitude of water level variations $3 * 10^{-3}[\mathrm{~Pa}]$ as the meteorological effect (Fig. 7). Periods of infrasound signals as well as of meteorological effect are similar and amount to tens of minutes. If we assume the identical coefficient of compensation for harmonic infrasounds as for meteorological effects i.e. $(99.99 \%)$ the harmonic infrasounds ought to be perfectly compensated in the space of corridors and not to affect the WT tiltmeter. This suggests that the coefficient of compensation of infrasound signals is very different from the coefficient of compensation of meteorological effects. Otherwise, infrasound signals in the atmosphere ought to be unacceptable large and would be visible in ordinary meteorological measurements. In fact we do not know what is the magnitude of investigated infrasound signals in atmosphere. The WT measurements provide us with information about the magnitude of infrasound signals multiplied by the coefficient of compensation which is unknown.

The works on harmonic infrasound signals will be continued. 


\section{REFERENCES}

Benioff, H. and Gutenberg, B.: 1939, Waves and currents recorded by electromagnetic barographs. Bull. Am. Met. Soc., 20, 421.

Donn, W.L. and Naini, B.: 1973, Sea wave origin of microbaroms and microseisms. J. Geophys. Res., 78 (21), 4482-4488. DOI: 10.1029/JC078i021p04482

Hoult, D.P.: 1969, Acoustic waves in the ionosphere, Pennsylvania State University Scientific Report No. 339.

Huba, J. D. and Joyce, G.: Ion sound waves in the topside low latitude ionosphere Plasma Physics Division, Naval Research Laboratory, Washington, D.C.J. A. Fedder, Leading Edge Technology, Washington, D.C. scientific letter.

Kaczorowski, M.: 2004, Water tube tiltmeter in Low Silesian Geophysical Observatory. Results of adjustment of half yearly series of plumb line variations, Acta Geodyn. Geomater., 1, No. 3 (135), 155-159.

Kaczorowski, M.: 2006A, Earth free oscillations observed in plumb line variations from the 26 December 2004 Earthquake., Acta Geodyn. Geomater., 3, No. 3 (143), 79-84.

Kaczorowski, M.: 2006B, High-Resolution Wide-Range Tiltmeter: Observations of Earth Free Oscillations Excited by the 26 December 2004 Sumatra -Andaman Earthquake. Monograph: - Earthquake Source Asymmetry, Structural Media and Rotation Effects. pp. 493-520, Springer-Verlag Berlin Heidelberg.

Ponomarev, E.A. and Sorokin, A.G.: 1996, Infrasonic waves in the atmosphere over east Siberia. Institute of SolarTerrestrial Physics SD RAS.,

Posmentier, E.S.: 1967, A theory of microbaroms, Geophys. JR Ast. Soc 13, 487-501.

Wenzel, H.-G.: 1996, The nanogal software: Earth tide data processing package ETERNA 3.30. Bulletin d'Informations Marees Terrestres, 124, 9425-9439, Bruxelles. 